\title{
Redefinição de limites territoriais em unidades de conservação na Amazônia brasileira: o caso da Floresta Nacional do Jamanxim, estado do Pará, Brasil \\ Redefinition of territorial limits of a conservation unit in the Brazilian Amazon: the case of the Jamanxim National Forest, state of Pará, Brazil
}

\author{
Paula Fernanda Viegas Pinheiro', Orleno Marques da Silva Júnior"l, Gilberto de Miranda Rocha"II, \\ Maria de Lourdes Pinheiro RuivoIv, Maria de Nazaré Martins Maciel', Thaís Gleice Martins Bragal, \\ Oberdan Oliveira Ferreira ${ }^{\text {III }}$ \\ 'Universidade Federal Rural da Amazônia. Belém, Pará, Brasil \\ "Universidade Federal do Rio de Janeiro. Rio de Janeiro, Rio de Janeiro, Brasil \\ IIIUniversidade Federal do Pará. Belém, Pará, Brasil \\ ıM Museu Paraense Emílio Goeldi/MCTIC. Belém, Pará, Brasil
}

\begin{abstract}
Resumo: A Floresta Nacional (FLONA) do Jamanxim foi criada em 2006, com 1.301.683,04 hectares, no município de Novo Progresso, estado do Pará. Desde a sua criação, tem sido palco de conflitos e de interesses econômicos, sendo pleiteada a (re)configuração territorial dela. $\bigcirc$ objetivo deste artigo é analisar de que forma esses conflitos vêm ocorrendo e quais as medidas que estão sendo tomadas pelos agentes locais e pelo poder público pela redefinição territorial da FLONA. As análises foram realizadas através da participação da autora no Grupo de Trabalho da Secretaria de Estado de Meio Ambiente do Pará (SEMA-PA), no qual foram ouvidos os agentes locais e os analistas do Instituto Chico Mendes de Conservação da Biodiversidade (ICMBio). Realizou-se análise por imagens de satélites e outras do Relatório do ICMBio, verificando-se que somente três das áreas mapeadas deveriam ser excluídas da FLONA, o que não foi aceito pelos agentes locais. Este fato foi gerador da medida provisória 756/2016, vetada pelo presidente da República, por contrariedade ao interesse público e inconstitucionalidade. Notadamente, há intensos conflitos e disputas por território, causando perdas no que concerne à conservação da biodiversidade, bem como diminuição do patrimônio natural da região amazônica.
\end{abstract}

Palavras-chave: Unidades de conservação. Biodiversidade. Geoprocessamento.

\begin{abstract}
The National Forest of Jamanxim in the municipality of Novo Progresso Pará state, Brazil, was created in 2006 with hectares, and since its creation has been the scene of conflicts among economic interests that have requested a redrawing of the national forest's boundaries. The purpose of this article is to analyze how these conflicts are occurring and the measures being taken by local agents and the government in the territorial redefinition of the national forest. Analyzes were carried out through the author's participation in a working group of the Pará state secretary for the environment in which both local agents and technicians of the Chico Mendes Institute for Biodiversity Conservation were heard Satellite image analysis and the ICMBio report were analyzed whereby only three of the mapped areas were to be excluded from the national forest, which was not accepted by the local agents, a triggering factor of provisional measure 756/2016, vetoed by the president of the Republic as contrary to public interests and unconstitutional. Notably, there are intense conflicts and disputes over territory, causing losses to the conservation of biodiversity and decrease of the natural heritage of the Amazon region.
\end{abstract}

Keywords: Forest reserve. Biodiversity. Geoprocessing.

PINHEIRO, P. F. V., O. M. SILVA JÚNIOR, M. L. P. RUIVO, M. N. M. MACIEL, T. G. M. BRAGA \& O. O. FERREIRA, 2017. Redefinição de limites territoriais em unidades de conservação na Amazônia brasileira: o caso da Floresta Nacional do Jamanxim, estado do Pará, Brasil. Boletim do Museu Paraense Emílio Goeldi. Ciências Naturais 12(3): 423-433.

Autora para correspondência: Paula Fernanda Viegas Pinheiro. Universidade Federal Rural da Amazônia. Instituto Ciberespacial. Av. Perimetral, 2501 - Terra Firme. Belém, PA, Brasil. CEP 66077-901 (paula.pinheiro@ufra.edu.br).

Recebido em 30/03/2017

Aprovado em 08/08/2017

Responsabilidade editorial: Fernando da Silva Carvalho Filho

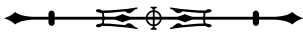




\section{INTRODUÇÃO}

A partir de 2005, com as altas taxas de desmatamento detectadas na Amazônia, o Brasil implementou um dos maiores programas de criação de áreas protegidas do mundo. Dados do Cadastro Nacional de Unidades de Conservação (CNUC) (MMA, 2016) mostram que, em 2016, as unidades de conservação (UC) cobriam 18,5\% do território brasileiro, o que corresponde a 158,4 milhões de hectares, sendo que $75 \%$ desse total estão no bioma Amazônia. Dessas áreas, 35\% correspondem ao grupo de proteção integral ${ }^{1}$ e $65 \%$ ao de uso sustentável ${ }^{2}$. Em 2016, o país abrigava, em seu território, cerca de 12\% das UC existentes no mundo (WDPA, 2016).

A criação destas áreas de conservação é extremamente positiva, pois, entre outros benefícios, garante a conservação da biodiversidade, bem como a manutenção do banco de germoplasma e do fluxo de água para o consumo humano (Medeiros et al., 2011), a conservação dos recursos naturais, tendo sido também um dos pilares da política de redução do desmatamento na região (Ferreira et al., 2005; MMA, 2011; Nolte et al., 2013; Stickler et al., 2013; Cohn et al., 2014; Araújo et al., 2015; Brandão Jr. et al., 2015).

Apesar de todas as vantagens anteriormente citadas, a expansão e a manutenção das áreas de conservação constituem potenciais conflitos com outros usos da terra, como agricultura, pecuária, exploração de madeira e obras de infraestrutura, que se tornam mais inflamáveis em regiões de expansão de ocupação, como é o caso da Amazônia.

Assim, as divergências de interesses colocam em conflito o aspecto ambiental-sustentável e o econômicocapitalista. Nessa perspectiva, o território, enquanto palco de construção e de desconstruções das relações socias, sofre alterações de acordo com o que rege a voz de comando, ordenando-se e desordenando-se conforme a necessidade social e econômica.
Diante desta realidade, este trabalho objetiva analisar o processo e os conflitos existentes na redefinição da Floresta Nacional do Jamanxim, estado do Pará, desde a sua criação, em 2006, até a sua redefinição, ocorrida em dezembro de 2016, para, assim, entender como os diferentes atores influenciaram nessa decisão e como essa redefinição pode afetar a conservação da natureza.

\section{ÁREA DE ESTUDO}

A Floresta Nacional (FLONA) do Jamanxim é uma unidade de conservação federal, de uso sustentável, criada pelo decreto de 13 de fevereiro de 2006, com uma área de 1.301.120 hectares, estando inserida inteiramente no município de Novo Progresso, no estado do Pará (Figura 1)

A FLONA é de posse e de domínio públicos, sendo que as áreas particulares incluídas em seus limites devem ser desapropriadas, de acordo com o que dispõe a lei 9.985/2000, que instituiu o Sistema Nacional de Unidades de Conservação (SNUC) no Brasil. Em 2008, quando da publicação do plano de manejo da unidade, constava que aproximadamente 2.700 habitantes viviam nas principais vicinais da FLONA e em seu entorno, muitos praticando atividades totalmente não condizentes com o objetivo da unidade, como agricultura, pecuária e exploração ilegal de madeira (ICMBio, 2008).

Entre os aspectos geoambientais existentes na região, a área que abrange a FLONA do Jamanxim apresenta geologia que sofreu influência da pediplanação pleistocênica, a qual isolou blocos de relevos residuais em litologias mais resistentes do paleo e mesoproterozoico, agrupados segundo características como a influência da tectônica regional sobre o relevo. A disposição dos blocos apresenta intensa dissecação, que não chega a atingir os níveis das depressões regionais, formando os planaltos residuais do sul do Pará (Brasil, 1973).

\footnotetext{
As áreas de proteção integral têm como função a manutenção dos ecossistemas livres de alterações causadas por interferência humanas, admitindo apenas o uso indireto dos seus atributos naturais (Brasil, 2000).

2 "O objetivo básico das unidades de uso sustentável é compatibilizar a conservação da natureza com o uso sustentável de parcela dos seus recursos naturais (Brasil, 2000).
}

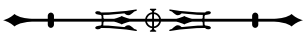




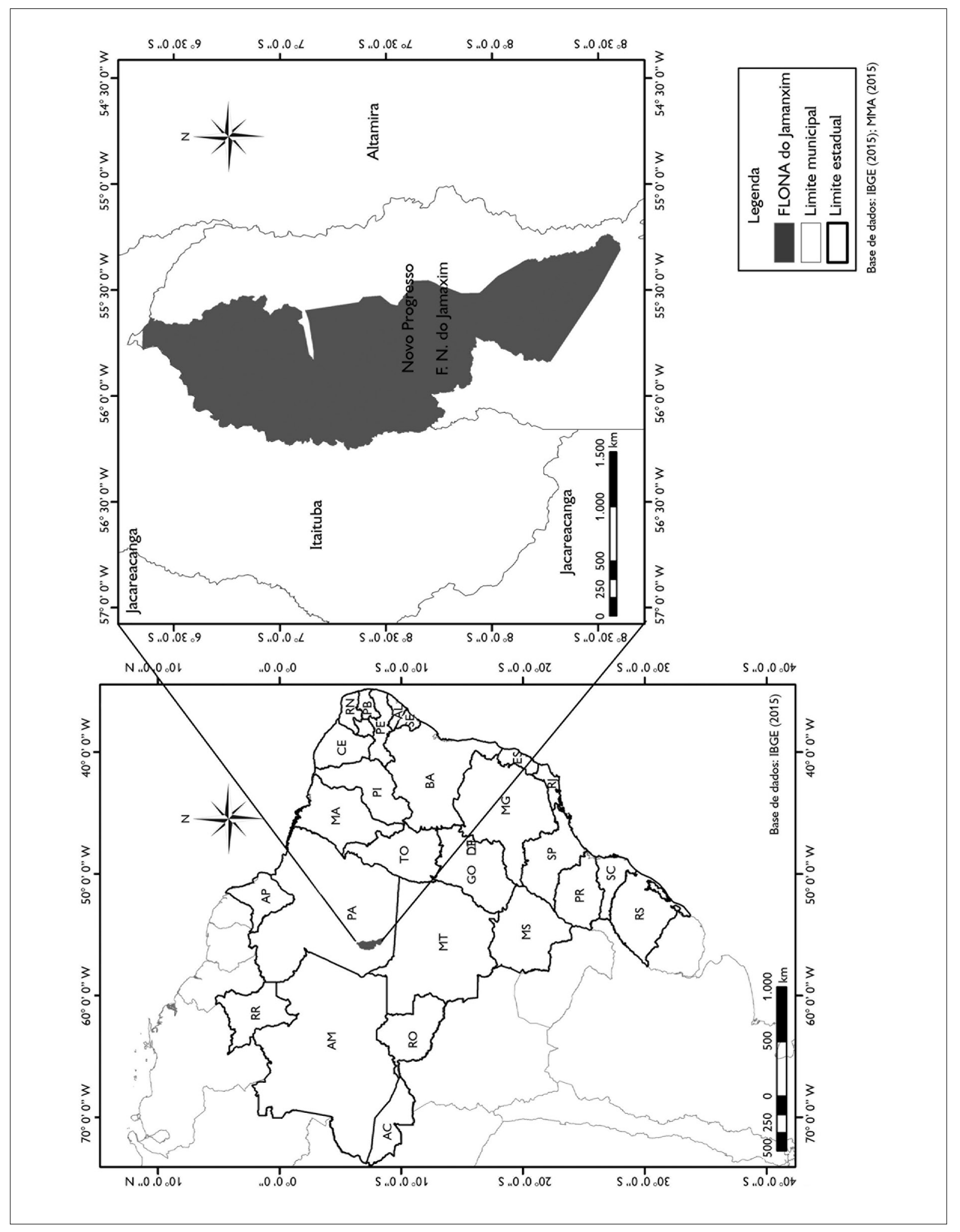

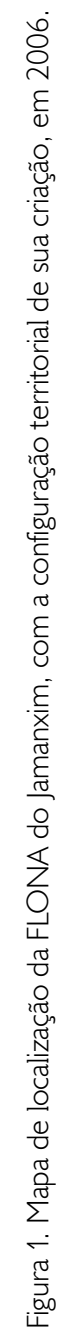

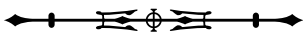


A criação da FLONA ocorreu em um contexto de ordenamento fundiário da área de influência da rodovia BR-163. Seu principal objetivo foi servir de barreira ao desmatamento e à exploração predatória e insustentável, que avançavam na região na mesma velocidade registrada no norte do Mato Grosso alguns anos antes, a qual é uma das regiões com maiores índices de desmatamento em toda a Amazônia brasileira, fato que se deve aos recursos naturais ali existentes e ao avanço da fronteira agropecuária.

Vem se observando que, ao longo da área de influência da BR-163, especificamente na porção em que se situa a FLONA do Jamanxim, ocorre o que se denomina de fenômeno de colonização espontânea, associado à colonização dirigida. Diz-se associada à colonização dirigida porque, apesar da ocupação espontânea e desordenada das terras da região, o processo de colonização contou com o apoio do Estado na construção de infraestrutura de transporte (Transamazônica e BR-163), através do Programa de Integração Nacional (PIN) e do Programa de Redistribuição de Terras e de Estímulo à Agroindústria do Norte e do Nordeste (PROTERRA), os quais incentivam a ocupação produtiva, o crédito agrícola subsidiado e outros subsídios fiscais, que influenciaram diretamente no aumento do fluxo migratório para a região nas décadas de 1970 e 1980.

\section{REDEFINIÇÃO DE UNIDADES DE CONSERVAÇÃO}

Não é somente no Brasil que as mudanças de limites em unidades de conservação são comuns; em várias partes do mundo (África, Ásia, América Latina e Caribe) são encontrados registros. Mascia et al. (2014) analisaram a ocorrência desses fenômenos, encontrando, entre 19002010, 595 casos de alterações em 395 áreas protegidas, ocorridas em 57 países. Segundo os autores, o evento mais frequente foi relacionado à mudança no tamanho das unidades de conservação (350 ou 60,8\% do total); em segundo lugar, observou-se a perda de proteção legal (150 casos ou 27,6\%); no seguimento, mudança de status de áreas protegidas para uma categoria de menor proteção, foram contabilizados 63 casos (11,6\%).

Quando se analisa as causas que levaram à redefinição das unidades de conservação, ainda segundo Mascia et al. (2014), estão associadas majoritariamente à necessidade de extração de recursos naturais em escala industrial. As questões fundiárias vêm logo sem seguida; e a revisão no sistema de unidades de conservação dos países aparece em terceiro lugar.

No Brasil, esses fenômenos também já foram alvo de estudos. Bernard et al. (2014) mostraram que, entre 1981 e 2012, registraram-se 93 eventos de redefinição de UC. No país, a principal causa desse fenômeno está relacionada à necessidade de expansão da geração e da transmissão de energia (Silva Jr. \& Santos, 2015).

Ao verificarem onde aconteceram essas mudanças, Silva Jr. \& Santos (2015) demonstraram que, dos 93 eventos, 69 ocorreram em UC de proteção integral e 24 em UC de uso sustentável. As UC gerenciadas pelos estados da federação apresentaram 68 casos, enquanto as de jurisdição federal, 25. Quando se considera o bioma, a Amazônia é onde predominam essas mudanças, 39 casos ou $42,3 \%$ do total.

O tipo de modificação mais comum são as reclassificações (49). Esse elevado número ocorreu após o advento da lei federal (9.985/2000) que estabeleceu o Sistema Nacional de Unidades de Conservação (SNUC), de maneira que as unidades pudessem se enquadrar nas categorias propostas pela nova legislação. Ocorreram ainda 26 com mudança de tamanho de área em UC, normalmente com perda de áreas, 11 revogações completas (extinção da UC) e cinco mudanças estão relacionados à diminuição de status de proteção (para uma categoria mais permissível).

\section{A (RE)CONFIGURAÇÃO TERRITORIAL DA FLONA JAMANXIM}

A delimitação territorial da FLONA do Jamanxim, desde a sua criação, foi alvo de grande discussão entre os atores sociais do município de Novo Progresso e do órgão gestor 
da unidade, o Instituto Chico Mendes de Conservação da Biodiversidade (ICMBio). Este fato manifesta-se ao se verificar que ambos concordam a respeito da necessidade de revisão dos limites definidos no decreto de criação da unidade, porém, apresentavam justificativas divergentes. Para tanto, trouxeram propostas, conjugadas e discutidas no embate de seus interesses, evidenciando como justificativa a otimização e a ocupação sustentável desse espaço territorial.

Nesse processo, desde 2009, foram feitas discussões e propostas por diversos segmentos da sociedade, entre eles, órgãos públicos de meio ambiente e associação de moradores de Novo Progresso, ou mesmo por parte de pessoas que habitavam o interior da FLONA, mas que praticavam atividades não condizentes com a restrição de uso da UC.

Foi criado um Grupo de Trabalho (GT) interdisciplinar, denominado de GT FLONA do Jamanxim, por meio do qual, em reunião realizada na SEMA-PA em novembro e dezembro de 2009, foi elaborada uma proposta de redefinição da FLONA, segundo a qual 46\% da área total permaneceriam com esta categoria de proteção, ou seja, quase 800 mil ha (300 mil ha menores em relação à área estipulada no decreto de criação), o que significa que cerca de 700 mil ha seriam pleiteados pela população local para exclusão (Figura 2) (SEMA, 2009).

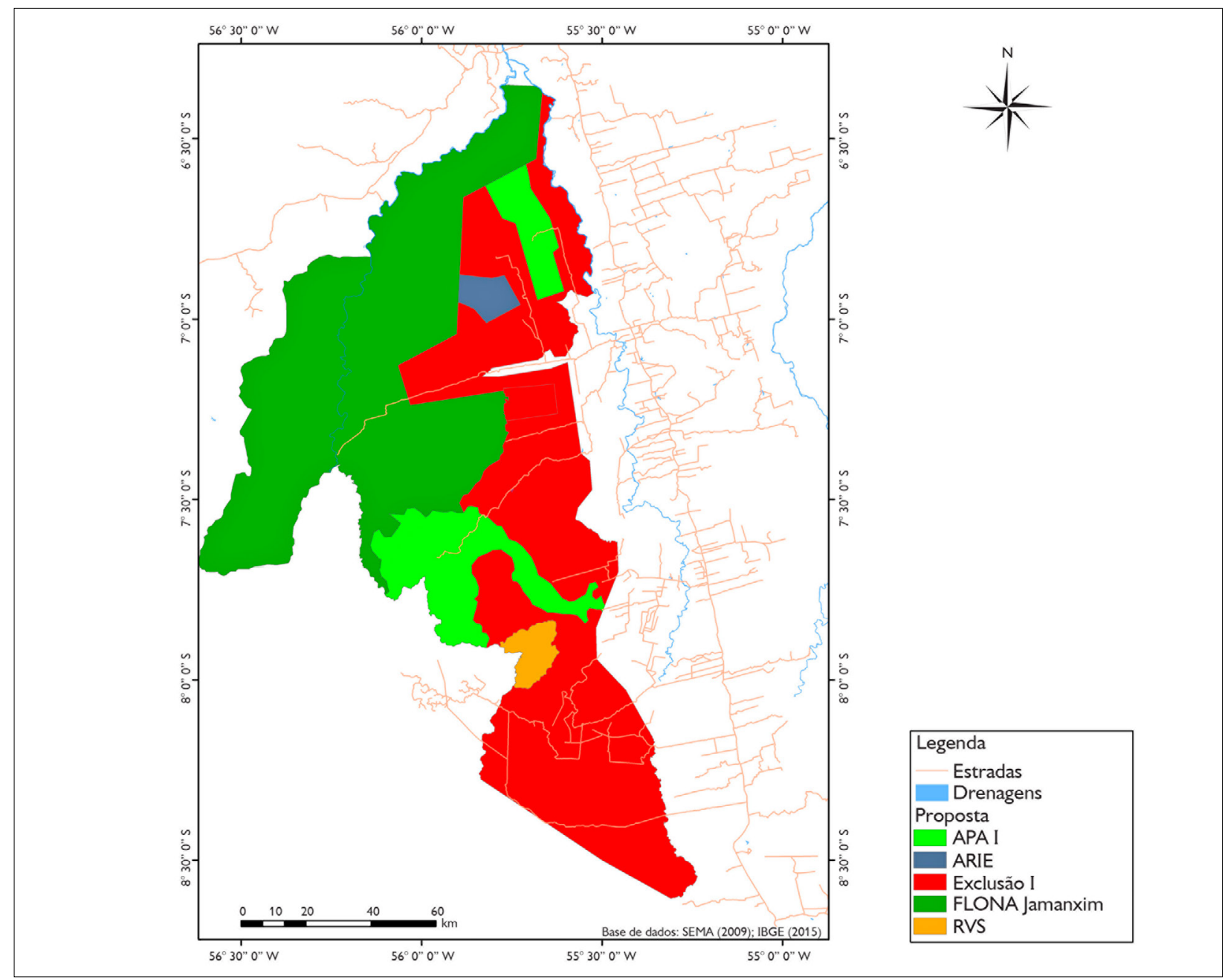

Figura 2. Configuração territorial proposta pelos atores sociais locais.

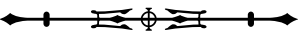


Durante as discussões do GT, foi manifestado que as áreas desafetadas não precisavam ser aquelas já ocupadas, mas também as necessárias para garantir o processo de desenvolvimento sustentável da ocupação regional.

A proposta colocada por alguns agentes locais dizia respeito à elaboração de limites/contornos para a formação de um mosaico de unidade de conservação na região da FLONA, quais sejam: FLONA do Jamanxim, Exclusão I e II, Área de Proteção Ambiental I, II e III, Refúgio da Vida Silvestre e Área de Relevante Interesse Ecológico. Tratase da criação e da modificação de categorias, divididas em pequenas outras áreas e que excluiriam quase completamente o limite original da UC.

Após a elaboração de uma proposta de redefinição territorial da FLONA pelos atores locais, o ICMBio apresentou o resultado de um trabalho que gerou o relatório intitulado "Estudo técnico de revisão dos limites da Floresta Nacional do Jamanxim" (ICMBio, 2009), segundo o qual a área da FLONA foi dividida em 13 áreas ou regiões, selecionadas por critérios técnicos dos analistas do instituto, que realizaram estudo de campo no período de 9 a 14 de setembro de 2009 (Figura 3).

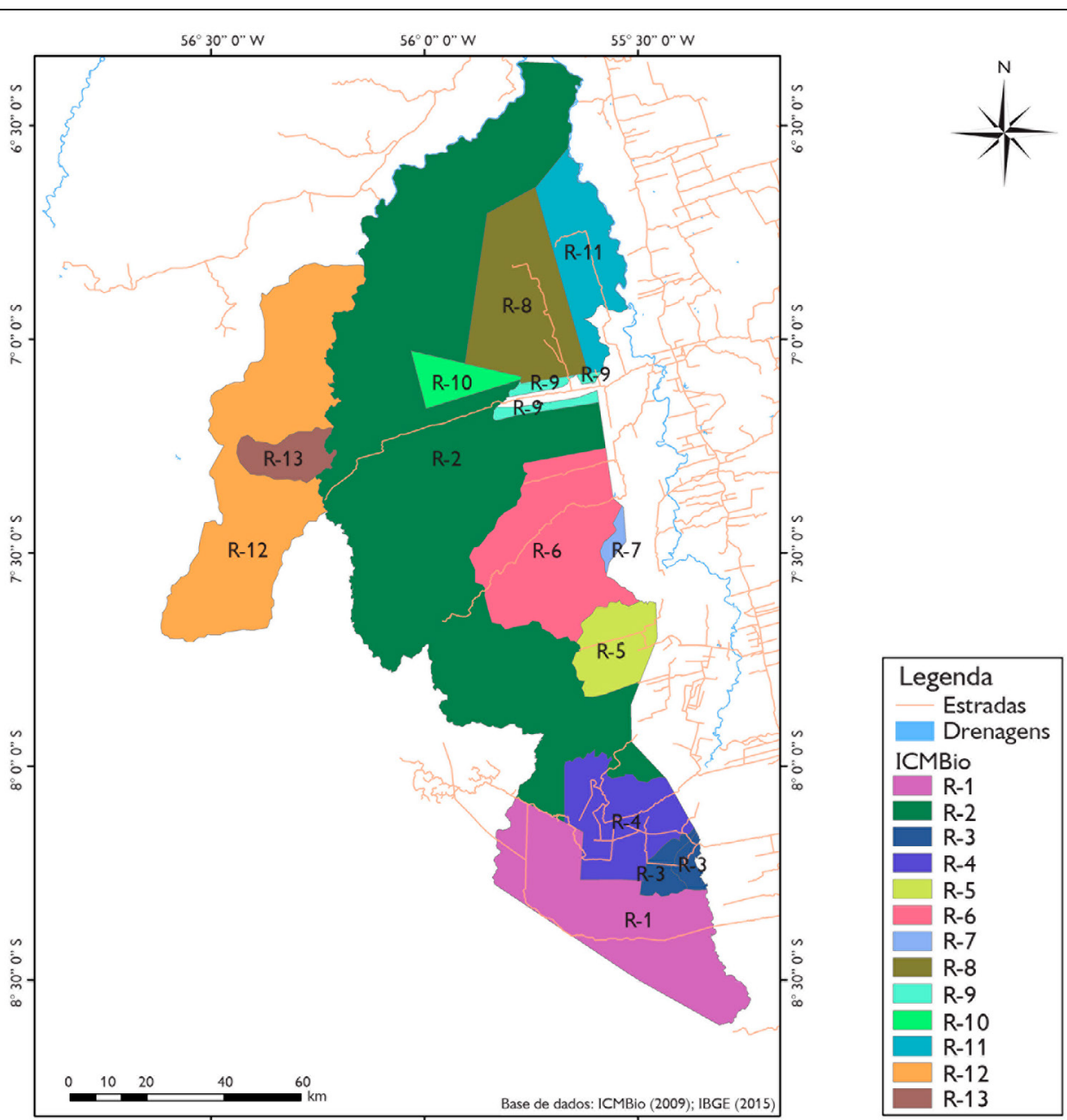

Figura 3. Configuração territorial proposta pelo ICMBio.

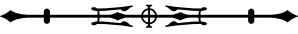


Destas áreas, o ICMBio somente considerou como propícias para exclusão da FLONA as que comprovassem maior ocupação, maior importância socioeconômica e baixo potencial para se manter dentro da unidade. Neste caso, somente seriam retiradas as áreas 3, 7 e 9 (Figura 3).

Em termos gerais, as comunidades locais, os atores sociais envolvidos e o ICMBio guardavam muita insatisfação em relação ao processo de reconfiguração territorial da FLONA Jamanxim, já que os interesses são divergentes. Isto alimentou grande receio entre estes envolvidos em relação ao futuro da FLONA diante da possibilidade de conservar, de forma sustentável, os recursos naturais ali presente, com o legado da política de ordenamento territorial.

Vale ressaltar que, além das vertentes econômicas, políticas e culturais, deve-se também considerar a vertente da natureza, que sempre estará presente dentro do território. A natureza, indissociavelmente, está no território (Pinheiro, 2010). Os seus recursos naturais, em função da grande busca e da valorização econômica deles), vêm a atender às exigências do mercado, além de estarem diretamente relacionados às alterações nos componentes físicos, químicos e biológicos dos territórios empreendidos (Pinheiro, 2010). Desse modo, esses elementos configuram-se como atrativos mercantis, para, assim, transformar o espaço em um território usado com a presença de um determinado poder, sendo este capaz de gerar implicações socioambientais (Haesbaert, 2002).

Trata-se da ocorrência dos impactos ambientais negativos, que podem ocasionar choques na dinâmica social, econômica e ambiental de um território. É necessário entender que, para formar o território, o espaço precisa existir e que a relação espaço e território se dá pela intensidade das técnicas e da diferenciação destes elementos, já que os espaços não são homogêneos (Santos, 2002).

Pinheiro (2010) ressalta que é no aparelhamento do território que serão verificadas as conexões de dominação a partir do uso destes elementos e de forças da sociedade. Desse modo, o uso intenso dos recursos naturais presentes na FLONA deixa transparecer os interesses, políticos, sociais, ambientais e, principalmente, empresariais, reproduzindose nesse espaço uma concentração de opulência natural, emparelhada a diferentes níveis de poder.

O objetivo básico de (re)ordenar os territórios ricos em recursos naturais, como ocorre no caso da FLONA do Jamanxim, deve ser o de oportunizar, em longo prazo, o uso múltiplo e sustentável dos recursos florestais e a pesquisa científica, com ênfase no desenvolvimento de métodos para exploração sustentável de florestas nativas.

Dessa forma, havia duas propostas de redefinição territorial para a FLONA do Jamanxim: a) dos agentes locais, segundo a qual a FLONA passaria a ter 781,380 ha; b) do ICMBio, que propunha um acréscimo de proteção de cerca de 1.514,856 ha de área protegida sob a forma de floresta nacional (Figura 4). Os estudos de redefinição territorial, tanto dos atores locais como do ICMBio, resultaram na configuração territorial instituída pela medida provisória (MP) n. 756/2016.

Como observado na Figura 4, as propostas de redefinição territorial da Floresta Nacional do Jamanxim eram totalmente opostas em termos de proteção da conservação da biodiversidade. Os agentes locais, partindo de seus próprios interesses, propunham uma redução drástica no tamanho da unidade de conservação, diminuindo-a em cerca de $60 \%$, tendo como consequência a obtenção de áreas livres para implantação de agricultura mecanizada, plantação e expansão da pecuária extensiva de corte e leiteira, além do aumento indiscriminado do desmatamento na região.

Por outro lado, o ICMBio propunha aumento em torno de $16 \%$ em relação ao tamanho original da FLONA, abrangendo, assim, parte do que hoje é a Área de Proteção Ambiental (APA) do Tapajós, com o intuito de aumentar a proteção, já que a APA é uma UC bem mais permissível. Essa forma ousada de extensão da UC, apesar de aumentar a proteção, significaria envolver outros atores e interesses, neste caso, os moradores da APA do Tapajós, que não aceitavam de nenhuma forma a mudança de categoria para outra de maior restrição. 


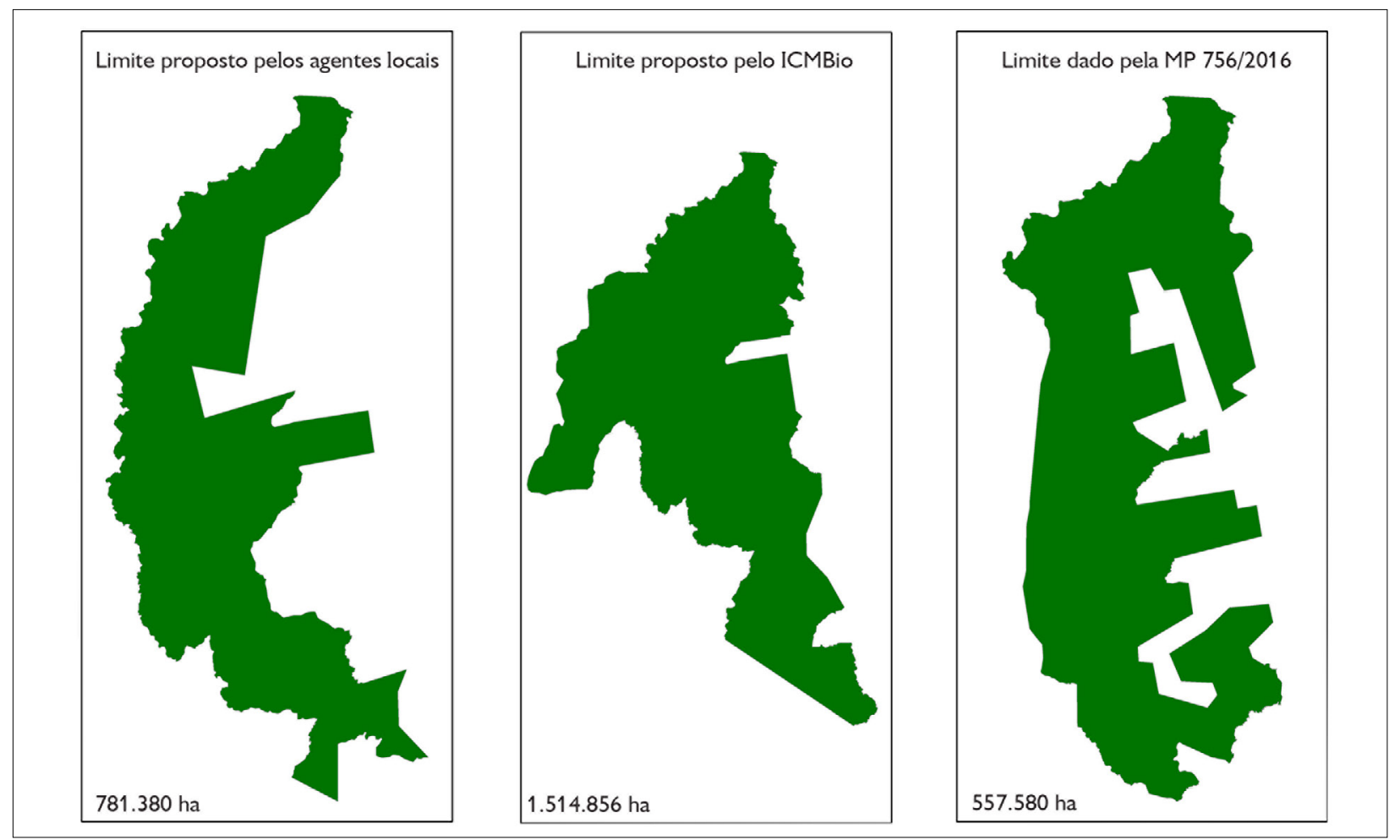

Figura 4. Síntese das três propostas para redefinição da FLONA do Jamanxim.

Percebe-se, nestes casos, que são vários os jogos de interesses e as formas de redefinição territorial, o que já frisava Pinheiro (2010), segundo a qual, independentemente da configuração territorial, o órgão gestor dessa unidade deveria ter recursos para gerir o território da UC, de forma que a mesma cumprisse seu papel fundamental, isto é, a proteção da biodiversidade.

Por fim, a redefinição dos limites ocorreu por meio da medida provisória 756, publicada em dezembro de 2016. $\mathrm{Na}$ exposição de motivos enviada ao presidente da República e assinada pelo Ministro do Meio Ambiente (Sarney Filho, 2016), abordou-se a redefinição dos limites da Floresta Nacional do Jamanxim, cujas alterações implicaram também mudança de limite do Parque Nacional (PARNA) do Rio Novo e criação da Área de Proteção Ambiental Jamanxim.

No texto da medida provisória, evidencia-se que os motivos principais da redefinição da FLONA estariam vinculados ao fato de:
O quadro geral é dominado pelo desenvolvimento de atividades econômicas informais, baseados na extração ilegal de madeira, minérios e grilagem de terra, com grande capacidade de alto financiamento. Neste cenário, as ações de comando e controle utilizadas não têm se mostrado suficientes para frear o avanço do desmatamento e a degradação dos corpos hídricos na região. $\bigcirc$ acirramento dos conflitos envolvendo os órgãos ambientais, tanto na gestão das áreas protegidas, como na execução das ações de comando e controle com as populações locais e infratores, é um reflexo desta situação, como resultado observa-se uma escalada na violência contra agentes públicos, com o aumento de confrontos armados, emboscadas e, infelizmente, mortes (Brasil, 2016).

Pelo que se pode depreender do texto, a gestão da UC é prejudicada por esses problemas de ocupação e pela realização de atividades ilegais, inclusive limitando as ações do poder público, principalmente no que tange às ações de proteção da área e o ao possível agravamento dos conflitos, devido ao advento de obras de infraestrutura,

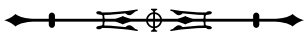


como o asfaltamento da BR-163 e a construção da ferrovia EF-170 (Ferrogrãos).

Houve, então, grande pressão política dos ocupantes, que pediam a revogação da FLONA; outros pediam uma recategorização para uma tipologia menos restritiva. No texto da MP 756/2016, foram excluídos da FLONA 304.772 hectares, recategorizados para uma APA, notadamente o tipo mais permissível de UC do SNUC.
No caminho inverso ao da diminuição do nível de proteção, mais 438.768 ha foram excluídos da FLONA e incorporados ao PARNA do Rio Novo, unidade de proteção integral com maior restrição de uso (Figura 5). No processo desencadeado pela MP 756/2016, também foi excluída uma área de 51.135 ha da APA do Tapajós, incorporada ao PARNA do Jamanxim, assim como foram excluídos 862 ha, destinados às faixas de domínio da estrada de ferro 170 e da BR-163.

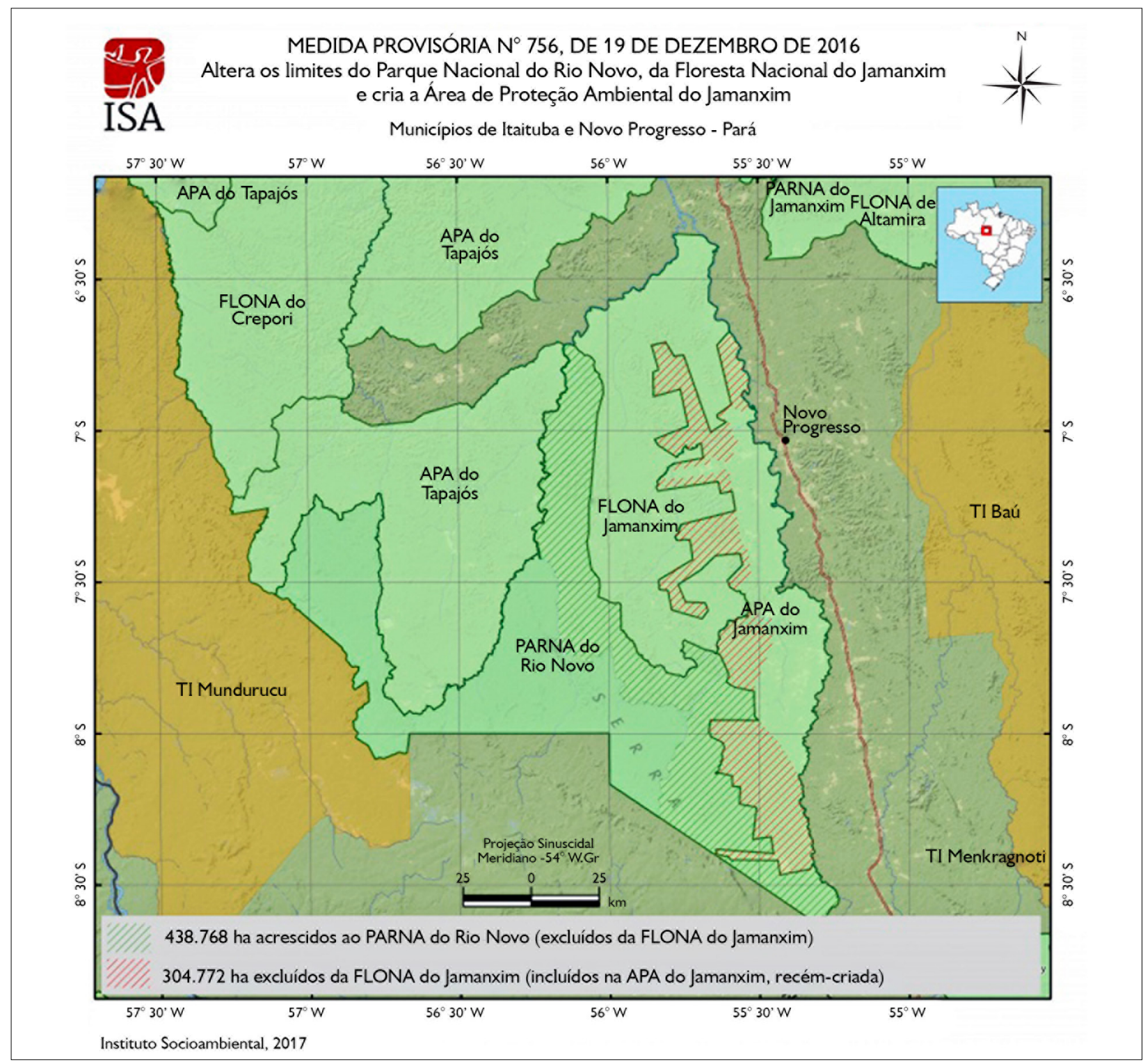

Figura 5. Alteração na FLONA do Jamanxim pela MP 756, municípios de Itaituba e Novo Progresso, Pará. Fonte: ISA (2016).

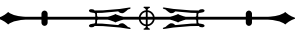


Ao observar a configuração atual mostrada na Figura 5 e as sínteses de propostas apresentadas na Figura 4, observa-se que claramente predominou o interesse dos agentes locais no processo de redefinição da UC, não levando em conta a posição do órgão gestor, ICMBio, que defendia não somente a manutenção da FLONA, como o aumento de sua área.

Aexclusão de cerca de 300 mil hectares deárea protegida abre precedentes para outras unidades de conservação na Amazônia, que também sofrem pressão antrópica.

No entanto, através da mensagem No 198, de 19 de junho de 2017, há a decisão de vetar integralmente, por contrariedade ao interesse público e inconstitucionalidade, a MP 756/201, tendo como principal argumento o de que "as modificações propostas alteram substancialmente o regime de proteção das unidades de conservação, com potencial de comprometer e fragilizar a preservação ambiental em uma região sensível da Amazônia brasileira" (Brasil, 2016).

Desta feita, a Floresta Nacional do Jamanxim continua com seu tamanho original conforme foi criada em 2006, mas se mantém como alvo de intensos conflitos pelo uso de seu território. Ainda terão muitos capítulos na história dessa importante unidade de conservação da Amazônia brasileira.

\section{CONSIDERAÇÕES FINAIS}

Pelos apontamentos do estudo realizado, mesmo admitindo-se a possibilidade de alguns ajustes nos atuais limites da FLONA do Jamanxim, qualquer revisão dos limites desta unidade deveria contemplar as correções de possíveis desvios ocorridos no ato de sua criação, como inclusão de áreas sem potencial para conservação ou já muito afetadas em termos antrópicos e exclusão de áreas com alto potencial de conservação e de uso sustentável dos recursos naturais.

É evidente, ainda, que as justificativas do poder público que motivaram a criação da FLONA do Jamanxim continuam vivas, buscando na região da BR-163 uma ocupação mais ordenada e responsável do que a que ocorreu no arco do desmatamento. Quaisquer alterações que descaracterizem a configuração atual da FLONA do Jamanxim, o recuo ou a desafetação de áreas causam prejuízos ambientais que perpassam além de seus limites geográficos.

Dessa forma, para que o (re)ordenamento territorial da FLONA do Jamanxim seja fortalecido, faz-se necessária adoção de medidas para que, concomitante à criação, à manutenção e à ampliação das terras protegidas, seja formado um conjunto de ações entre o poder público e a sociedade organizada, por meio de instrumentos como a regularização fundiária, incentivos fiscais e creditícios para a concessão de florestas públicas, a fim de serem manejadas, com o objetivo de aumentar a eficiência econômica e a sustentabilidade dessas áreas.

Não menos importante é a implementação de obras de infraestrutura sustentáveis; a geração de emprego e de renda em atividades de recuperação de áreas degradadas; bem como a incorporação de áreas abertas e abandonadas ao processo produtivo, através da atuação de forma integrada dos órgãos municipais, estaduais e federais, sendo os mesmos responsáveis pelo monitoramento e a intensificação da fiscalização contra desmatamento ilegal, priorizando a qualidade do manejo das já escassas florestas nacionais.

Neste artigo, é defendida a ideia de que as unidades de conservação apresentam um legado enquanto instrumentos de ordenamento ambiental, desde que seus objetivos sejam alcançados, isto é, a conservação da biodiversidade a que estão propostos ou o controle que seus limites físicos impõem ao uso ilegal de seus recursos naturais protegidos nestes locais.

\section{REFERÊNCIAS}

ARAÚJO, E., P. BARRETO \& H. MARTINS, 2015. Áreas protegidas críticas na Amazônia no período de 2012 a 2014: 1-20. Ed. Imazon, Belém.

BERNARD, E., L. PENNA \& E. ARAUJO, 2014. Downgrading, downsizing, degazettement, and reclassification of protected areas in Brazil. Conservation Biology 28(4): 939-950. DOI: <http://dx.doi. org/10.1111/cobi.12298>.

BRANDÃO JR., A., P. BARRETO, C. SOUZA JR. \& B. BRITO, 2015. Evolução das emissões de gases de efeito estufa no Brasil (19902013) - setor de mudança de uso da terra: 1-29. Observatório do Clima, São Paulo. 
BRASIL, 1973. Projeto RADAMBRASIL: Folha SA 23, geologia, geomorfologia, solos, vegetação e uso potencial da terra. Programa de Integração Nacional, Rio de Janeiro.

BRASIL, 2000. Lei n. 9.985, de 18 de julho de 2000. Regulamenta o art. 225, § 1o, incisos I, II, III e VII da Constituição Federal, institui o Sistema Nacional de Unidades de Conservação da Natureza e dá outras providências. Diário Oficial da União, 19 julho 2000. Disponível em: <http://www.mma.gov.br/port/conama/legiabre. $\mathrm{cfm}$ ?codlegi=322>. Acesso em: janeiro 2017.

BRASIL, 2016. Medida provisória n. 756, de 19 de dezembro de 2016. Altera os limites do Parque Nacional do Rio Novo, da Floresta Nacional do Jamanxim e cria a Área de Proteção Ambiental do Jamanxim. Diário Oficial da União, 20 dezembro 2016. Disponível em: < http:// www2.camara.leg.br/legin/fed/medpro/2016/medidaprovisoria-75619-dezembro-2016-784061-exposicaodemotivos-151720-pe.html>. Acesso em: janeiro 2017.

COHN, A. S., A. MOSNIER, P. HAVLIK, H. VALIN, M. HERRERO, E. SCHMID, M. O'HARE \& M. OBERSTEINER, 2014. Cattle ranching intensification in Brazil can reduce global greenhouse gas emission by sparing land from defloretation. Proceedings of the National Academy of Sciences of the United States of America 111(20): 7236-7241. DOI: <http://dx.doi.org/10.1073/pnas.1307163111>.

FERREIRA, L., E. VENTICINQUE \& S. ALMEIDA, 2005. O desmatamento na Amazônia e a importância das áreas protegidas. Estudos Avançados 19(53): 157-166. DOI: <http://dx.doi. org/10.1590/S0103-40142005000100010>.

HAESBAERT, R., 2002. Territórios alternativos. Contexto, São Paulo.

INSTITUTO CHICO MENDES DE CONSERVAÇÃO DA BIODIVERSIDADE (ICMBio), 2008. Planos de manejo das Florestas Nacionais do Jamanxim, Crepori e do Amana, no Estado do Pará. Relatório do diagnóstico socioeconomico da FLONA do Jamanxim. ICMBio, Brasília.

INSTITUTO CHICO MENDES DE CONSERVAÇÃO DA BIODIVERSIDADE (ICMBio), 2009. Estudo técnico de revisão dos limites da Floresta Nacional do Jamanxim. Relatório final. ICMBio, Brasilia.

INSTITUTO SOCIOAMBIENTAL (ISA), 2016. Governo Temer altera Unidades de Conservação e fragiliza proteção à floresta no Pará. Notícias Sociambientais, 21 dez. 2016. Disponível em: <https:// www.socioambiental.org/pt-br/noticias-socioambientais/governotemer-altera-unidades-de-conservacao-e-fragiliza-protecao-a-florestano-para > . Acesso em: fevereiro 2017.

MASCIA, M., S. PAILLER, R. KRITHIVASAN, V. ROSHCHANKA, D. BURNS, M. MLOTHA, D. MURRAY \& N. D. PENG, 2014. Protected area downgrading, downsizing, and degazettement (PADDD) in Africa, Asia, and Latin America and the Caribbean, 1900-2010. Biological Conservation 169: 355-361. DOI: <https://doi.org/10.1016/j. biocon.2013.11.021>.
MEDEIROS, R., C. YOUNG, H. PAVESE \& F. ARAÚJO, 2011. Contribuição das unidades de conservação brasileiras para a economia nacional: sumário executivo: 1-44. UNEP-WCMC, Brasília.

MINISTÉRIO DO MEIO AMBIENTE (MMA), 2011. Análise do desmatamento nas unidades de conservação federais localizadas na Amazônia. ICMBio. Disponível em: < http:// www.mma.gov.br/estruturas/182/_arquivos/icmbio_ucs_182. pdf $>$. Acesso em: dezembro 2015.

MINISTÉRIO DO MEIO AMBIENTE (MMA), 2016. Cadastro Nacional de Unidades de Conservação Disponível em: < http:// www.mma.gov.br/areas-protegidas/cadastro-nacional-de-ucs $>$. Acesso em: dezembro de 2016.

NOLTE, C., A. AGRAWAL, K. SILVIUS \& B. SOARES-FILHO, 2013. Governance regime and location influence avoided deforestation success of protected areas in the Brazilian Amazon. Proceedings of the National Academy of Sciences 110(13): 4956-4961. DOI: <http://dx.doi.org/10.1073/ pnas.1214786110>.

PINHEIRO, P. F. V. , 2010. A cartografia e o geoprocessamento como instrumentos de análise das propostas de redefinição dos limites territoriais da Floresta Nacional do Jamanxim - Estado do Pará. Dissertação (Mestrado em Geografia) Universidade Federal do Pará, Belém.

SANTOS, M., 2002. Territórios: globalização e fragmentação. Hucitec/Annablumme, São Paulo.

SARNEY FILHO, José, 2016. EM n 00071/2016 MMA. Disponível em: <http://www.planalto.gov.br/ccivil_03/_ato20152018/2016/exm/exm-mp-756-16.pdf> . Acesso em: janēiro 2017.

SECRETARIA DE ESTADO DE MEIO AMBIENTE (SEMA), 2009. Relatório técnico do resultado das reuniões dos atores sociais sobre a redefinição da FLONA do Jamanxim. SEMA, Belém.

SILVA JR., O. \& M. SANTOS, 2015. Redefinição de limites de áreas protegidas para produção de energia na Amazônia. Cadernos de Energia (2): 1-59.

STICKLER, C., M. COE, M. COSTA, D. NEPSTAD, D. MCGRATH, L. DIAS, H. RODRIGUES \& B. SOARES FILHO, 2013. Dependence of hydropower energy generation of forest in the Amazon Basin at local and regional scales. Proceedings of the National Academy of Sciences 110(23): 9601-9606. DOI: $<$ http://dx.doi.org/10.1073/pnas.1215331110>.

WORLD DATABASE ON PROTECTED AREAS (WDPA), 2016. Statistic UNEP/WCMC/IUCN/WCPA. Disponível em: <https:// www.iucn.org/theme/protected-areas/our-work/quality-andeffectiveness/world-database-protected-areas-wdpa $>$. Acesso em: janeiro de 2016.

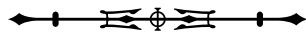


